\title{
Dualität bei Steuerungsproblemen und zugeordneten Flußproblemen I
}

\author{
R. KLÖTZLER
}

In dieser Arbeit werden neuartige Flußprobleme vorgestellt, die als spezielle ,, reląxed problems" zu stetigen Steuerungsproblemen mit Funktionen von einer Variablen in Erscheinung treten. Beide Optimierungsaufgaben besitzen jeweils das gleiche Dualproblem, die Eigenschaft der starkeñ Düalität läßt sich bei den Flußproblemen-aber unter geringeren Voraussetzungenbeantworten.

В настоящей работе исследуется связь между непрерывными задачами оптимального управления одной переменной и специальво построенными потоковыми задаqами. Двойственные вадачи этих проблем совпадают, а сильную двойственность можно получить для потоковой задачи при более слабых условиях.

In this paper novel classes of relaxed problems are stated which are called flow problems. They are related to standard problems of optimal control with functions of one independent variable. Both of these optimization problems have in each case the same dual problem. However for flow problems one can prove the validity of strong duality under weaker assump. tions.

\section{Einleitung}

In einer Vorlesungsreihe „Duality in Optimal Control“ am Banachzentrum Warschau im Herbst 1980 hat der Verfasser zu homogenen Variationsproblemen $\left(P_{h}\right)$ einfacher Integrale in Parameterdarstellung ein allgemeines Dualitätsprinzip vorgestellt. Es beinhaltet die Konstruktion einer dualen Optimierungsaufgabe $\left(D P_{h}\right)$, deren Dualproblem $\left(D P_{h}\right)^{*}$ im Sinne von R. T. Rockafelcar ein Flußproblem darstellt, das mit $\left(P_{h}\right)$,,verwandt" ist, indem es als ein verschmiertes Problem (, ,relaxed problem") zu $\left(P_{h}\right)$ aufgefaßt werden kann. Während bei $\left(P_{h}\right)$ nur quellenfreie Flüsse in Gestalt von Trajektorien der „Dicke“ Null zugelassen werden, sind zulässige Elemente von $\left(D P_{h}\right)^{*}$ demgegenüber durch beliebige richtungşbeschränkte quellenfreie Flüsse vorgegebener Intensität gekennzeichnet. Im einzelnen sind Untersuchungen dazu in [6] dargelegt worden sowie Bedingungen, unter denen zwischen $\left(D P_{h}\right)$ und $\left(D P_{h}\right)^{*}$ starke Dualität nachweisbar ist. Diese für praktische Abschätzungen von Optimalwerten nützliche Dualität soll nachstehend nunmehr auch für allgemeine Steuerprobleme einfacher Integrale (in inhomogener Darstellung) aufgebaut werden. Es lassen sich dabei Ergebnisse erzielen, die über den Youngschen Flußbegriff [10] in enger Beziehung zu einschlägigen Dualitätsaussagen von $R$. B. VINTER und $R$. $M$. LEwIS [9] stehen, ohne jedoch mit diesen identisch zu sein. 


\section{Steuerungsprobleme einfacher Integrale}

Wir studieren Steuerungsprobleme des Typs

$$
J_{0}(x, u):=\int_{0}^{T} f_{0}(t, x(t), u(t)) d t \rightarrow \inf
$$

bezüglich aller $x \in W_{1}{ }^{1}, n(0, T), u \in L_{1}{ }^{l}(0, T)$, unter den Zustandsgleichungen $\dot{x}=g(t, x, u)$, Zustandsbeschränkungen $x(t) \in X(t) \subset \mathbf{E}^{n} \quad \forall t \in[0, T]$,

Steuerbeschränkungen $u(t) \in U(t, x(t))$ für fast alle $t \in[0, T]$ und den Randbedingungen

$$
. x(0) \in \mathfrak{M}_{0} \subset X(0), \quad x(T) \in \mathfrak{M}_{T} \subset X(T) .
$$

Wir stellen dabei folgende Grundvoraussetzungen:

1. $G=\left\{(t, \xi) \in \mathbf{E}^{1} \times \mathbf{E}^{n} \mid \xi \in X(t), t \in(0, T)\right\}$ ist ein Lipschitzgebiet des $\left.\mathbf{E}^{n+1}{ }^{1}\right)$.

2. $U(\cdot, \cdot)$ ist eine normale mengenwertige Abbildung von $\bar{G}$ in den $\mathbf{E}^{l}$ (im Sinne von [3]) und für sämtliche $x \in W_{1}^{1, n}(0, T)$ mit $x(t) \in X(t)$ auf $[0, T]$ sei $U(\cdot, x(\cdot))$ eine normale mengenwertige Abbildung von $[0, T]$ in den $\left.\mathbf{E}^{(} .^{2}\right)$

3. die $U(t, \xi)$ sind gleichmäßig beschränkt im $\mathbf{E}^{\imath}$ für alle $(t, \xi) \in \bar{G}$.

4. $\mathfrak{M}_{0}$ und $\mathfrak{M}_{T}$ sind Lipschitzgebiete des $\mathbf{E}^{n} ; f_{0}$ und $g$ sind stetig auf $\bar{G} \times \mathbf{E}^{n}$.

5. die Menge $\mathfrak{P}$ aller zulässigen Prozesse $(x, u)$ von $(1)$ sei nicht leer.

Dann gilt nach R. KLötzler [4] und C. P. ORtLIeb [7] folgender Dualitätssatz.

Satz 1: $J_{0}(x, u) \geqq L(S):=S_{T}-S_{0}$ für alle $(x, u) \in \mathfrak{B}$ und beliebige $S \in W_{\infty}^{1}(G)$, welche mit $\mathfrak{M}_{0}{ }^{*}:=\{0\} \times \mathfrak{M}_{0}$ und $\mathfrak{M}_{T}{ }^{*}:=\{T\} \times \mathfrak{M}_{T}$ auf $G$ den Nebenbedingungen

$$
\begin{aligned}
& \nabla S(t, \xi) \in \widetilde{\mho}_{0}(t, \xi)= \\
& \left\{\left(z_{0}, z_{1}{ }^{\prime}\right) \in \mathbf{E}^{1} \times \mathbf{E}^{n} \mid \forall v \in U(t, \xi) \quad \text { ist } \quad z_{0}+z_{1}{ }^{\prime} g(t, \xi, v) \leqq f_{0}(t, \xi, v)\right\}
\end{aligned}
$$

genügen (fast überall) und auf $\mathfrak{M}_{0}{ }^{*}$ und $\mathfrak{M}_{T}{ }^{*}$ konstante Werte $S_{0}$ bzw. $S_{T}$ besitzen. ${ }^{3}$ )

Die Menge der in Satz 1 zulässigen Funktionen $S$ bezeichnen wir mit $\mathfrak{S}$. $\mathfrak{F}_{0}(t, \xi)$ nennen wir Figuratrixkörper zu Problem (1) an der Stelle $(t ; \xi) \in G$. $\mathfrak{\mho}_{0}(t, \xi)$ ist offensichtlich stets eine konvexe Menge des $\mathbf{E}^{n+1}$. Die Aufgabe

$$
L(S) \rightarrow \sup \quad \text { auf } \subseteq
$$

stellt eine duale Aufgabe zu (1) dar in der allgemeinen Auffassung von [4]. Utber eine ökonomische Interpretation dieser Dualität vgl. [7]. Wegen der Konvexität aller $\mathfrak{F}_{0}(t, \xi)$ ist $(3)$ ein konvexes Optimierungsproblem mit linearem Zielfunktional $L$ bezüglich $S$.

In Anlehnung an [8, S. 188] und [9, S. 550] kann das Steuerungsproblem (1) leicht auch durch ein äquivalentes Variationsproblem ersetzt werden. Wir stiutzen uns dabei auf folgenden Hilfssatz.

1) Im Sinne von C. B. Morrey bzw. S. Hildebrandt: Uber die Identität der Sobolewscihen und der Calkin-Morreyschen Räume. Math. Ann. 148 (1962), 226-237.

2) Hinreichende Bedingungen für diese Eigenschaft werden im Anhang I angegeben.

$\left.{ }^{3}\right)$ Nach [4] ist diese Aussage zunächst nur für solche $S$ gesichert, die zugleich $\operatorname{dem} C^{\mathbf{1}}(\bar{G})$ angehören; in Analogie zu [5] läßt sich diese Fassung leicht aber auch auf den Dualitätssatz obiger Art erweitern, vgl. dazu Anhang II. 
Hilfssatz 1: Es sei für alle $(t, \xi) \in \bar{G}$ und $z \in V(t, \xi)$

mit

$$
f(t, \xi, z):=\operatorname{Min}_{v}\left\{f_{0}(t, \xi, v) \mid z=g(t, \xi, v), v \in U(t, \xi)\right\}
$$

$$
V(t, \xi):=\left\{\zeta \in \mathbf{E}^{n} \mid \zeta=g(t, \xi, v), v \in U(t, \xi)\right\} .
$$

Dann ist das Variationsproblem

$$
J(x):=\int_{0}^{T} f(t, x(t), \dot{x}(t)) d t \rightarrow \inf \text { auf } \mathfrak{X}
$$

mit $\mathfrak{X}:=\left\{x \in W_{1}^{1, n}(0, T) \mid(t, x(t)) \in \bar{G}\right.$ auf $[0, T]$,

$$
\begin{aligned}
& \dot{x}(t) \in V(t, x(t)) \text { fast überall auf }[0, T], \\
& \left.x(0) \in \mathfrak{M}_{0}, x(T) \in \mathfrak{M}_{T}\right\}
\end{aligned}
$$

äquivalent zu (1). Das heißt, es ist $\underset{\mathfrak{i}}{\dot{i}} J=\inf _{\mathfrak{F}} J_{0}$.

Beweis: Wir bemerken zunächst, daß infolge unserer Grundvoraussetzungen (2) gemäß $[3, \S 8.1]$ für sämtliche $x \in \mathfrak{X}$ der Integrand in (6) tatsächlich auch summierbar ist. Im weiteren beweisen wir die erwähnte Ãquivalenz.

a) Es sei $\left\{\left(x_{n}, u_{n}\right)\right\}$ eine Minimalfolge zu (1). Dann ist nach (4)

$$
f(t, \xi, g(t, \xi, v)) \leqq f_{0}(t, \xi, v) \forall v \in U(t, \xi) \text { und }(t, \xi) \in \bar{G} .
$$

Deshalb ist

$$
\begin{aligned}
\underset{\mathfrak{B}}{\inf } J_{0} & =\lim _{n \rightarrow \infty} \int_{0}^{T} f_{0}\left(t, x_{n}(t), u_{n}(t)\right) d t \\
& \geqq \underset{n \rightarrow \infty}{\lim _{n}} \int_{0}^{T} f\left(t, x_{n}(t), g\left(t, x_{n}(t), u_{n}(t)\right)\right) d t \\
& =\lim _{n \rightarrow \infty} \int_{0}^{T} f\left(t, x_{n}(t), \dot{x}_{n}(t)\right) d t \geqq \inf _{\mathfrak{x}} J .
\end{aligned}
$$

b) Es sei $\left\{x_{n}\right\}$ eine Minimalfolge zu (6). Dann ist nach [3, S. 289 Folgerung 2] die mengenwertige Abbildung

$$
\begin{aligned}
& t \rightarrow \psi_{n}(t):= \\
& . \quad\left\{v \in U\left(t, x_{n}(t)\right) \mid \dot{x}_{n}(t)=g\left(t, x_{n}(t), v\right), f_{0}\left(t, x_{n}(t), v\right)=f\left(t, x_{n}(t), \dot{x}_{n}(t)\right)\right\}
\end{aligned}
$$

infolge unserer Grundvoraussetzungen (2) normal, so daß für jedes $n$ ein meßbarer Schnitt $u_{n}$ existiert mit

$$
u_{n}(t) \in U\left(t, x_{n}(t)\right), \dot{x}_{n}(t)=g\left(t, x_{n}(t), u_{n}(t)\right),
$$

$\dot{f}_{0}\left(t, x_{n}(t), u_{n}(t)\right)=f\left(t, x_{n}(t), \dot{x}_{n}(t)\right)$ für fast alle $t \in[0,7]$. Wegen der gleichı̈äßigen Beschränktheit aller $U(t, \xi)$ auf $G$ sind sämtliche $u_{n}$ beschränkt und summierbar.

Deshalb ist auch

$$
\begin{aligned}
\underset{\mathfrak{i} f}{\inf } & =\lim _{n \rightarrow \infty} \int_{0}^{T} f\left(t, x_{n}(t), \dot{x}_{n}(t)\right) d t \\
& =\lim _{n \rightarrow \infty} \int_{0}^{T} f_{0}\left(t, x_{n}(t), u_{n}(t)\right) d t \geqq \underset{\mathfrak{S}}{\geqq} \inf _{0} .
\end{aligned}
$$


In Zusammenfassung beider Ergebnisse von a) und b) resultiert die Behauptung von Hilfssatz 1

Satz 2: $J(x) \geqq L(S)=S_{T}-S_{0}$ fïr alle $x \in \mathfrak{X}$ und $S \in \mathfrak{S}$.

Beweis: Nach Satz 1, angewandt auf das spezielle Steuerungsproblem (6), gilt zunächst der Dualitätssatz $J(x) \geqq L(S) \forall x \in \mathfrak{X}$ und $S \in W_{\infty}^{1}(G)$, welche auf $G$ fast überall den Nebenbedingungen

$$
\begin{aligned}
& \nabla S(t, \xi) \in \mathfrak{F}(t, \xi):= \\
& \left\{\left(z_{0}, z_{1}^{\prime}\right) \in \mathbf{E}^{1} \times \mathbf{E}^{n} \mid \forall v \in V(t, \xi) . \text { ist } \quad z_{0}+z_{1}{ }^{\prime} w \leqq f(t, \xi, w)\right\}
\end{aligned}
$$

genügen und auf $\mathfrak{M}_{0}{ }^{*}$ und $\mathfrak{M}_{T}{ }^{*}$ jeweils konstante Werte $S_{0}$ bzw. $S_{T}$ annehmen. Zum Beweis von Satz 2 genügt es folglich lediglich noch zu zeigen, daß für alle $(t, \xi) \in \bar{G}$ stets $\mathfrak{F}_{0}(t, \xi)=\mathfrak{F}(t, \xi)$ ist.

Es sei $\left(z_{0}, z_{1}^{\prime}\right) \in \mathfrak{F}(t, \xi)$. Dann ist gemäß (5) für $v \in U(t, \xi)$ auch $g(t, \xi, v) \in V(t, \xi)$, so da $\cap$ aus $z_{0}+z_{1}^{\prime} w \leqq f(t, \xi, w) \forall w \in V(t, \xi)$ wegen (4) speziell

$$
z_{0}+z_{1}^{\prime} g(t, \xi, v) \leqq f(t, \xi, g(t, \xi, v)) \leqq f_{0}(t, \xi, v) \quad \forall v \in U(t, \xi)
$$

folgt. Das bedeutet $\left(z_{0}, z_{1}{ }^{\prime}\right) \in \mathfrak{F}_{0}(t, \xi)$ bzw. $\mathfrak{F}(t, \xi) \subset \mathfrak{F}_{0}(t, \xi)$.

Ist umgekehrt $\left(z_{0}, z_{1}^{\prime}\right) \in \mathfrak{F}_{0}(t, \xi)$, so gilt

$$
z_{0}+z_{1}{ }^{\prime} g(t, \xi, v) \leqq f_{0}(t, \xi, v) \quad \forall v \in U(t, \xi) .
$$

Deshalb ist wegen (4) und (5)

$$
z_{0}+z_{1}^{\prime} g(t, \xi, v) \leqq f(t, \xi, g(t, \xi, v)) \quad \forall v \in U(t, \xi)
$$

bzw. mit $w=g(t, \xi, v)$

$$
z_{0}+z_{1}{ }^{\prime} w \leqq f(t, \xi, w) \quad \forall w \in V(t, \xi) .
$$

Das bedeutet $\left(z_{0}, z_{1}{ }^{\prime}\right) \in \mathfrak{F}(t, \xi)$ bzw. $\mathfrak{F}_{0}(t, \xi) \subset \mathfrak{F}(t, \xi)$. In Zusammenfassung beider Úberlegungen resultiert daraus $\mathfrak{F}=\mathfrak{F}_{0}$

\section{Flußprobleme erster Art}

Die in den Sätzen 1 und 2 ausgedrückte Dualität der Optimierungsprobleme (1)/(3) bzw. $(\theta) /(3)$ ist nicht involutorisch. Wir erkennen dies schon an der Konstruktion des dualen Problems (3). Während in den Primalproblemen (1) bzw. (6) Zielfunktionale in Gestalt einfacher Integrale auftreten und zulässige Elemente als vektorwertige Funktionen von einer Veränderlichen, hat das Dualproblem (3) ein ganz anders geartetes Zielfunktional. Außderdem sind die zulässigen Elemente von (3) Funktionen von mehreren unabhängigen Variablen. Unsere Konstruktion eines Dualproblems zu (1) bzw. (6) läßt damit auch nicht erkennen, wie nun wiederum zu (3) ein duales Problem konstruiert werden kann. Diesen scheinbaren Nachteil werden wir dadurch überbrücken, indem wir dem Problem (6), und damit auch (1), ein Flußproblem zur Seite stellen, das sich im weiteren durch folgende Eigenschaften auszeichnet. Es entsteht einerseits durch „Verschmieren“ des Problems (6), indem wir an Stelle der zulässigen Trajektorien zu (6) (als „,fadenförmig dünne“. Flüsse) auch divergenzfreie Flüsse allgemeinerer Art zulassen. Dieses so entstehende Flußproblem wird das gleiche Dualproblem (3) besitzen. Schließlich wird sich dieses Flußproblem zugleich als duales Problem zu (3) im Sinne der speziellen Fenchel- 
Rockafellarschen Dualität erweisen. Aus methodischen Gründen führen wir ein solches zugeordnetes Flußproblem in zwei Etappen ein, als Flußproblem erster bzw. zweiter Art.

Wir betrachten das Optimierungsproblem

$$
J_{1}(\mathfrak{v}):=\int_{G} f(t, \xi, v(t, \xi)) v_{0}(t, \xi) d t d \xi \rightarrow \inf
$$

bezüglich aller

$$
\mathfrak{b}=v_{0} \cdot(1, v) \in C^{1, n+1}(\bar{G})
$$

mit den Eigenschaften

$$
\begin{aligned}
& v_{0}(t, \xi) \geqq 0, \quad v(t, \xi) \in V(t, \xi) \text { fast überall auf } G ; \\
& \nabla \cdot \mathfrak{v}=0 \text { auf } G ; \\
& \int_{\mathfrak{R}_{0}} v_{0}(0, \xi) d \xi=1, \quad \int_{\mathfrak{R}_{T}} v_{0}(T, \xi) d \xi=1 ; \\
& \left.\mathfrak{v} \cdot d o=0 \text { auf } \partial G_{0}:=\partial G \backslash\left(\mathfrak{M}_{0}^{*} \cup \mathfrak{M}_{T}{ }^{*}\right) \cdot{ }^{4}\right)
\end{aligned}
$$

Indem wir auch hier wieder die Grundvoraussetzungen (2) übernehmen, ist nach $[3, \S 8.1]$ die Funktion $f(\cdot, \cdot, v(\cdot, \cdot))$ summierbar auf $G$ sogar für beliebige summierbare $v$; außerdem ist $V(\cdot, \cdot)$ gemä $B(4)$ eine normale mengenwertige Abbildung auf $G$. Wir bezeichnen (8) in bezug auf (6) (oder (1)) als das zugeordnete Flu $\beta$ problem erster Art. Seine zulässigen Elemente $\mathfrak{v}$ nennen wir Flüsse, ihre Gesamtheit bezeichnen wir mit $\mathfrak{B}$.

Satz 3: Das Optimierungsproblem (3) ist ein duales Problem zum Flußproblem (8).

Beweis: Für beliebige $\mathfrak{v} \in \mathfrak{B}$ und $S \in \mathcal{S}$ ist wegen (8d) zunächst

$$
J_{1}(\mathfrak{v})=\int_{G}\left[f(t, \xi, v(t, \xi)) v_{0}(t, \xi)+S(t, \xi) \nabla \cdot \mathfrak{v}\right] d t d \xi
$$

Wegen $\nabla \cdot(S(t, \xi) \mathfrak{v}(t, \xi))=\nabla S(t, \xi) \cdot \mathfrak{v}(t, \xi)+S(t, \xi) \nabla \cdot \mathfrak{v}(t, \xi)$ können wir unter Anwendung des Gaußschen Integralsatzes für (9) auch schreiben

$$
\begin{aligned}
J_{1}(\mathfrak{v})= & \int_{G}\left[f(t, \xi, v(t, \xi)) v_{0}(t, \xi)-\nabla S(t, \xi) \mathfrak{v}(t, \xi)\right] d t d \xi \\
& +\int_{\partial G} S(t, \xi) \mathfrak{v}(t, \xi) d o .
\end{aligned}
$$

Infolge $\mathfrak{v}=v_{0} \cdot(1, v) \in \mathfrak{B}$ ist nach $(8 \mathrm{c}) v_{0} \geqq 0$ und wegen $S \in \subseteq$ zugleich $\nabla S(t, \xi)$ $\epsilon \mathfrak{F}_{0}(t, \xi)=\mathfrak{F}(t, \xi)$. Das bedeutet nach $(7) f(t, \xi, v) v_{0}(t, \xi)-\nabla S(t, \xi) \cdot \mathfrak{v}(t, \xi) \geqq 0$ und, eingesetzt in (10), wegen (8e) und (8f)

$$
J_{1}(\mathfrak{v}) \geqq S_{T}-S_{0} \quad \forall \mathfrak{v} \in \mathfrak{B} \quad \text { und } \quad S \in \mathbb{S} .
$$

Diese Eigenschaft kennzeichnet Problem (3) als eine duale Optimierungsaufgabe zu (8)

4) do fassen wir hier und im weiteren stets vektoriell als orientiertes Oberflächenelement von $\partial G$ auf. 


\section{Flußprobleme zweiter Art}

Die Differenzierbarkeitsforderungen $(8 b)$ an $\mathfrak{v}$ stellen Einschränkungen dar, denen bei vielen realen Problemen die dort vorkommenden Flüsse nicht genügen. Denken wir z. B. nur daran, innerhalb $G$ von einem Ort $\mathfrak{M}_{0}{ }^{*}$ nach einem zweiten Ort $\mathfrak{M}_{r}{ }^{*}$ eine Flüssigkeit möglichst billig abzuleiten, so wird mit der optimalen Leitungsführung ein Vektorfluß erzeugt, der außerhalb des Leitungsrohres abrupt (unstetig) in den Fluß der Intensität Null übergeht. Aus diesem Grunde erweitern wir nachstehend die Zielstellung (8a) auf eine größere Klasse von Flüssen $\mathfrak{b}$, 'die nur in distributivem Sinne die Bedingungen ( $8 \mathrm{~d})$ und ( $8 \mathrm{f}$ ) erfüllen. Wir führen dazu ein:

$$
\Xi_{0}:=\left\{S \in W_{\infty}^{1}(G) \mid S=\text { const je auf } \mathfrak{M}_{0}^{*} \text { und } \mathfrak{M} \mathfrak{R}_{T}^{*}\right\} \text {. }
$$

Abkürzend bezeichnen wir wiederum $\left.S\right|_{\mathfrak{M}_{0^{*}}}=S_{0}$ und $\left.S\right|_{\mathfrak{m}_{T^{*}}}=S_{T \cdot}$. Versehen mit der Topologie des Banachraumes $W_{\infty}^{1}(G)$ ist $\mathfrak{\subseteq}_{0}$ daron ein Unterraum und somit selbst ein Banachraum. Weiterhin erklären wir

$$
\begin{aligned}
& \mathfrak{W}:=\left\{\mathfrak{v} \in L_{1}{ }^{n+1}(G) \mid \mathfrak{v}=v_{0} \cdot(1, v), v_{0}(t, \xi) \geqq 0, v(t, \xi) \in V(t, \xi)\right. \\
& \left.\int_{G} \nabla S(t, \xi) \mathfrak{v}(t, \xi) d t d \xi=S_{T}-S_{0} \quad \forall S \in \mathcal{S}_{0}\right\} \text {. }
\end{aligned}
$$

Wir bezeichnen dann die Aufgabe

$$
J_{2}(\mathfrak{v}):=\int_{G} f(t, \xi, v(t, \xi)) v_{0}(t, \xi) d t d \xi \rightarrow \inf \quad \text { auf } \mathfrak{W} \because
$$

in bezug auf (6) (oder (1)) als das zugeordnete Flußproblem zweiter Art.

Hilfssatı 2: $\mathfrak{B} \subset \mathfrak{W}$.

Beweis: Für $\mathfrak{v} \in \mathfrak{B}$ ist wegen $(8 b)$ nach dem Gaußschen Intcgralsatz für alle $S \in \widetilde{S}_{0}$

$$
\begin{aligned}
\int_{G} \nabla S(t, \xi) \mathfrak{v}(t, \xi) d t d \xi= & -\int_{G} S(t, \xi) \nabla \cdot \mathfrak{v}(t, \xi) d t d \xi \\
& +\int_{\partial G} S(t, \xi) \mathfrak{v}(t, \xi) d o .
\end{aligned}
$$

Da außerdem nach Voraussetzung die Bedingungen (8d)-(8f) gelten, folgt aus (15)

$$
\begin{aligned}
& \int_{C} \nabla S(t, \xi) \mathfrak{v}(t, \xi) d t d \xi \\
& =-\int_{\mathscr{R _ { 0 }}} S(0, \xi) v_{0}(0, \xi) d \xi+\int_{\mathbb{N}_{T}} S(T, \xi) v_{0}(T, \xi) d \xi=S_{T}-S_{0} \quad \forall S \in \Subset .
\end{aligned}
$$

In Verbindung mit (8c) besagt dieses Ergebnis (16), daß für alle $\mathfrak{v} \in \mathfrak{B}$ stets $\mathfrak{v} \in \mathfrak{W}$ gilt bzw. $\mathfrak{B} \subset \mathfrak{W}$ :

Hilfssatz 3: $\mathfrak{B} \cap C^{1, n+1}(\vec{G}) \subset \mathfrak{B}$.

Beweis: Es sei $\mathfrak{v} \in \mathfrak{W} \cap C^{1, n+1}(\bar{G})$. Dann gilt für dieses wiederum Formel (15) für beliebige $S \in \mathfrak{S}_{0}$. Wählen wir speziell $S$ willkürlich aus $\dot{W}_{\infty}^{1}(\bar{G})$, so folgt aus $(1 \overline{5})$ wegen $\mathfrak{v} \in \mathfrak{M}$ die Variationsgleichung

$$
\int_{G} S(t, \xi) \nabla \cdot \mathfrak{v}(t, \xi) d t d \xi=0 \quad \forall S \in \mathfrak{W}_{\infty}^{\mathfrak{l}}(G)
$$

Und weil $\mathscr{W}_{\infty}^{1}(G)$ dicht in $L_{1}(G)$ ist, resultiert aus (17) das Ergebnis $\nabla \cdot \mathfrak{v}(t, \xi)=0$ auf $G$, womit (8d) erfüllt ist. 
Setzen wir in (15) unter Beachtung des vorangehenden Resultats beliebige $S \in \mathfrak{S}_{0}$ ein, erhalten wir wegen $\mathfrak{v} \in \mathfrak{M}$

$$
\begin{aligned}
S_{T}-S_{0}= & \int_{\partial G} S(t, \xi) \mathfrak{v}(t, \xi) d o \\
= & \int_{\partial G_{0}} S(t, \xi) \mathfrak{v}(t, \xi) d o+\int_{\mathscr{\mathfrak { V } _ { T }}} S(T, \xi) v_{0}(T, \xi) d \xi \\
& -\int_{\mathfrak{D} t_{0}} S(0, \xi) v_{0}(0, \xi) d \xi .
\end{aligned}
$$

Speziell für $S \in \mathcal{S}_{0}$ mit der Eigenschaft $S_{T}=S_{0}$ folgt aus (18)

$$
\int_{\partial C_{0}} S\left(t^{\prime}, \xi\right) \mathfrak{v}(t, \xi) d o=0 \quad \forall S \in \subseteq_{0} \mid S_{T}=S_{0}
$$

Dieses Resultat hat of fensichtlich $\mathfrak{v} d o=0$ auf $\partial G_{0}$ zur Folge, also Eigenschaft (8f). Unter Beachtung dieses neuen Resultats vereinfacht sich (18) zu

$$
S_{T}-S_{0}=S_{T} \int_{\mathfrak{M}_{T}} v_{0}(T, \xi) d \xi-S_{0} \int_{\mathbb{N}_{0}} v_{0}(0, \xi) d \xi \quad \forall S \in \mathfrak{S}_{0} .
$$

Wegen der Willkürlichkeit der Konstanten $S_{0}$ und $S_{T} \operatorname{muß}$ somit $\int_{\mathfrak{R}_{T}} v_{0}(T, \xi) d \xi=1$ und $\int_{\mathscr{D} \text { 。 }} v_{0}(0, \xi) d \xi=1$ sein, also Eigenschaft $(8 \mathrm{e})$ gelten. Da die Elemente von $\mathfrak{W}$ außerdem per Definition (8c) erfüllen, ist schließlich $\mathfrak{v} \in \mathfrak{B}$ für alle $\mathfrak{v} \in \mathfrak{W B} \cap C^{1, n+1}(\bar{G})$; damit ist Hilfssatz 3 bewiesen $B$

Satz 4: Das Optimierungsproblem (3) ist auch ein duales Problem zum Flußproblem (14).

Beweis: Für beliebige $\mathfrak{v} \in \mathfrak{W}$ und $S \in \mathfrak{S} \subset \mathfrak{S}_{0}$ gilt gemäß (13)

$$
J_{2}(\mathfrak{v})=\int_{G}\left[f(t, \xi, v(t, \xi)) v_{0}(t, \xi)_{1}-\nabla S(t, \xi) \mathfrak{v}(t, \xi)\right] d t d \xi+S_{T}-S_{0} .
$$

Wegen $S \in \mathfrak{S}$ ist $\nabla S(t, \xi) \in \mathfrak{F}_{0}(t, \xi)=\mathfrak{F}(t, \xi) \forall(t, \xi) \in G$, außerdem ist wegen $\mathfrak{v}=v_{0} \cdot(1, v) \in \mathfrak{M}$ zugleich $v_{0} \geqq 0$. Deshalb ist der Integrand des vorangehenden Ausdrucks nicht négativ und somit $J_{2}(\mathfrak{y}) \geqq S_{T}-S_{0}$. Diese Eigenschaft drückt die Dualität zwischen den Problemen (14) und (3) aus $\mathrm{B}$

\section{Bezichungen zur Rockafellarschen Dualität}

Wir sagen, die Optimierungsprobleme (14) und (3) stehen zueinander in starker Dualität, wenn

$$
\inf _{\mathfrak{B}} J_{2}=\sup _{\subseteq} L
$$

gilt. Unter welchen Bedingungen können wir diese Eigenschaft sichern? Wir benutzen zur Beantwortung dieser Frage Weiterführungen und Stabilitätsaussagen der Fenchel-Rockafellarschen 'Theorie der konjugierten Funktionale gemäß EkeLaNd/ Temam [1].

Hilfssatz 4: Das Optimierungsproblem (3) ist auch in Sinne der speziellen FenchelRockafellarschen Dualitätskonzeption ein duales Problem zum Flußproblem (14). 
Beweis: Die Fenchel-Rockafellarsche Konstruktion eines dualen Optimierungsproblems beruht auf folgenden Prinzip. Es seien $X$ und $Y$ zwei topologische Vektorräumo und $X^{*}$ bzw. $Y^{*}$ ihre dualen Räume. Weiterhin sei $\Phi$ ein reelles Funktional auf $X \times Y$ und

$$
\Phi(\mathfrak{b}, 0) \rightarrow \text { inf auf } X
$$

das Primalproblem. Dann ist das konjugierte Funktional $\Phi^{*}$ zu $\Phi$ im Sinne von Fenchel und Rockafellar bekanntlich definiert durch

$$
\Phi^{*}\left(q^{*}, p^{*}\right):=\sup _{\mathfrak{B} \in X, p \in Y}\left[\left\langle q^{*}, \mathfrak{v}\right\rangle+\left\langle p^{*}, p\right\rangle-\Phi(\mathfrak{v}, p)\right]
$$

für beliebige $\left(q^{*}, p^{*}\right) \in X^{*} \times Y^{*}$, wobei $\langle\cdot, \cdot\rangle$ das Produkt von Elementen dualer Räume ausdrückt. Die Aufgabe

$$
-\dot{\Phi}^{*}\left(0, p^{*}\right) \rightarrow \sup \text { auf } Y^{*}
$$

ist dann ein duales Problem zu (20).

Zur Bestätigung von Hilfssatz 4 werden wir zeigen, daß bei geeigneter Wahl von $X, Y$ und $\Phi$ diese Konstruktion zu (14) als Primalproblem das Problem (3) als duales Problem erzeugt. Dazu setzen wir $X=L_{1}{ }^{n+1}(G), Y=\mathfrak{S}_{0}{ }^{*}$ und versehen $Y$ mit der schwachen Topologie von $\mathfrak{S}_{0}{ }^{*}$. Daraus resultiert $Y^{*}=\mathfrak{S}_{0}^{* *}=\mathfrak{S}_{0}$ (vgl. z. B. [2]). Weiterhin führen wir einen linearen stetigen Operator $\Lambda \in \mathscr{L}(X \rightarrow Y)$ ein, welcher durch

$$
\langle S, A \mathfrak{v})=-\int_{G} \nabla S(t, \xi) \mathfrak{v}(t, \xi) d t d \xi \quad \forall S \in \mathfrak{S}_{0}
$$

definiert wird. Da die rechte Seite von (18) auch als $-\langle\nabla S, \mathfrak{v}\rangle$ geschrieben werden kann, ist $-\Lambda$ gleich dem adjungierten Operator $\nabla^{*}$ zu $\nabla . \Phi$ definieren, wir hier für beliebige $(\mathfrak{v}, p) \in X \times Y$ durch

$$
\Phi(\mathfrak{v}, p):=\left\{\begin{array}{ll}
J_{2}(\mathfrak{v}) & \text { für alle } \mathfrak{v}=v_{0} \cdot(1, v) \text { mit } v_{0} \geqq 0 \\
& v(t, \xi) \in V(t, \xi) \text { fast überall auf } G \\
& \text { und }\langle S, \Lambda \mathfrak{v}-p\rangle+S_{T}^{\prime}-S_{0}=0 \quad \forall S \in Y^{*} ; . \\
\infty & \text { andernfalls. }
\end{array} \quad \forall .\right.
$$

Damit tritt für $p=0$ der erste Fall in (24) genau dann auf, wenn $\mathfrak{v} \in \mathfrak{M}$ ist. Deshalb ist bei dieser Wahl von $\Phi$ das FluBproblem (14) äquivalent mit (20), indem

gilt.

$$
\inf _{\mathfrak{B}} J_{2}=\inf _{\mathfrak{v} \in X} \Phi(\mathfrak{v}, 0)
$$

Nunmehr berechnen wir $\Phi^{*}\left(0, p^{*}\right)$ über die Vorschrift (21). Dazu beachten wir, daß die in (24) benutzte Bedingung

$$
\langle S, A \mathfrak{v}-p\rangle+S_{T}-S_{0}=0 \quad \forall S \in Y^{*}
$$

$z u$ gegebenem $\mathfrak{v}$ in eindeutiger Weise eine Lösung $p \in Y$ dieser Variationsgleichung definiert. Diese von $\mathfrak{v}$ abhängige lösung bezeichnen wir mit $p=A \mathfrak{v}$. Damit wird

$$
\Phi^{*}\left(0, p^{*}\right)=\sup _{\mathfrak{v} \in X, p \in Y}\left[\left\langle p^{*}, p\right\rangle-\Phi(\mathfrak{v}, p)\right]=\sup _{\mathfrak{v} \in X, p=\mathcal{A}_{\mathfrak{v}}}\left[\left\langle p^{*}, p\right\rangle-\Phi(\mathfrak{b}, p)\right] .
$$

Weil $p=A \mathfrak{v}$ die Variationsgleichung (25) identisch befriedigt, folgt speziell mit $S=-p^{*}$ aus (25) das Resultat $\left\langle p^{*}, p\right\rangle=\left\langle p^{*}, \Lambda \mathfrak{v}\right\rangle+p_{T}^{*}-p_{0}^{*}$. Dies cingesetzt in (26) liefert

$$
\Phi\left(0, p^{*}\right)=\sup _{\mathfrak{v} \in X . p=A}\left[p_{T}^{*}-p_{0}^{*}+\left\langle p^{*}, \Lambda \mathfrak{v}\right\rangle-\Phi(\mathfrak{v}, p)\right]
$$


und mit (23) und (24)

$$
\begin{aligned}
\Phi^{*}\left(0, p^{*}\right)= & \sup _{\mathfrak{v} \in X_{0}}\left[p_{T}^{*}-p_{0}^{*}-\int_{G}\left(\nabla p^{*}(t, \xi) \mathfrak{v}(t, \xi)\right.\right. \\
& \left.\left.+v_{0}(t, \xi) f(t, \xi, v(t, \xi))\right) d t d \xi\right]
\end{aligned}
$$

wobei abkürzend $X_{0}:=\left\{\mathfrak{b} \in X \mid \mathfrak{v}=v_{0} \cdot(1, v), v_{0} \geqq 0, v(t, \xi) \in V(t, \xi)\right\}$ darstellt. Dies ergibt

$$
\Phi^{*}\left(0, p^{*}\right)= \begin{cases}p_{T}^{*}-p_{0}^{*}, & \text { wenn }-\nabla p^{*}(t, \xi) \in \mathfrak{F}(t, \xi) \text { fast } \\ & \text { überall auf } G \\ \infty & \text { sonst. }\end{cases}
$$

Mit diesem Resultat (27) wird die Identität des Problems (22) zu der nachstehenden Aufgabe offensichtlich. Diese lautet

$$
L\left(-p^{*}\right)=p_{0}^{*}-p_{T}^{*} \rightarrow \sup
$$

bezüglich aller $p^{*} \in \mathfrak{S}_{0}$ mit $-\nabla p^{*}(t, \xi) \in \mathfrak{F}(t, \xi)$ fast überall auf $G$. Setzen wir wieder $-p^{*}=S$ in (28) ein, so wird dieses Problem (28) sogar formal identisch mit (3), wenn wir noch die Eigenschaft $\mathfrak{F}(t, \xi)=\mathfrak{F}_{0}(t, \xi)$ aus Abschnitt 2 beachten. Damit ist Hilfssatz 4 bewiesen

Auf der Grundlage dieses Hilfssatzes lassen sich in Anwendung der Rockafellarschen Theorie Aussagen über starke Dualität entwickeln. Darüber wird in Teil II berichtet.

\section{Anhang I: Hinreichende Bedingungen zu den Grundvoranssetzungen (2)}

Hilfssatz 5 : Es sei $U_{0}(\cdot)$ eine normale mengenwertige Abbildung von $[0, T]$ in den $\mathbf{E}^{l}$ und $\chi$ eine Abbildung des $[0, T] \times \mathbf{E}^{n} \times \mathbf{E}^{l}$ in den $\mathbf{E}^{k}$ mit den Eigenschaften:

(i) $\chi(\cdot, \xi, v)$ ist meßbar auf $[0, T] \forall(\xi, v) \in \mathbf{E}^{n} \times \mathbf{E}^{l}$,

(ii) $\chi(t, \cdot, \cdot)$ ist stetig auf $\mathbf{E}^{n} \times \mathbf{E}^{l} \forall t \in[0, T]$.

Dann ist $U(\cdot, \cdot)$ gemä $\beta$

$$
U(t, \xi):=\left\{v \in U_{0}(t) \mid \chi(t, \xi, v) \leqq 0\right\}
$$

eine normale mengenwertige Abbildung von $[0, T] \times \mathbf{E}^{n}$ in den $\mathbf{E}^{l}$, und für sämlliche $x \in C^{0, n}[0, T]$ ist $U(\cdot, x(\cdot))$ eine normale mengenwertige Abbildung von $[0, T]$ in den $\mathbf{E}^{l}$.

Beweis: Nach [3, Kap. 8.1] ist $U_{0}$ genau dann eine meßbare mengenwertige Abbildung von $[0, T]$ in den $\mathbf{E}^{l}$, wenn $z u U_{0}$ eine approximierende Familie $\left\{u_{v}(\cdot)\right\}$ meBbarer Funktionen $u$, auf $[0, T]$ existiert. Setzen wir diese Funktionen $u_{v}$ auf ganz $[0, T] \times \mathbf{E}^{n}$ fort durch die Definition $\bar{u}_{v}(t, \xi)=u_{v}(t) \forall \xi \in \mathbf{E}^{n}$, so bildet die Familie $\left\{\bar{u}_{\nu}(\cdot, \cdot)\right\}$ zugleich eine approximierende Familie der mengenwertigen Abbildung $\bar{U}_{0}(\cdot, \cdot)$ mit $\bar{U}_{0}(t, \xi):=U_{0}(t) \forall \cdot \epsilon[0, T]$ und $\xi \in \mathbf{E}^{n}$. Folglich ist $\bar{U}_{0}(\cdot, \cdot)$ auch eine meßbare mengenwertige Abbildung von $[0, T] \times \mathbf{E}^{n}$ in den $\mathbf{E}^{\prime}$. Wenn $U_{0}$ außerdem normal ist, d. h. sämtliche $U_{0}(t)$ sind abgeschlossene Punktmengen des $\mathbf{E}^{l}$, so ist $\bar{U}_{0}$ offenbär auch normal. Damit ist $U(\cdot ; \cdot)$ auch darstellbar durch

$$
U(t, \xi)=\left\{v \in \bar{U}_{0}(t, \xi) \mid \not(t, \xi, v) \leqq 0\right\} .
$$

Hieraus entnehmen wir nach [3] (S. 289, Folgerung 2), daß $U(\cdot, \cdot)$ eine normale, mengenwertige Abbildung von $[0, T] \times \mathbf{E}^{n}$ in den $\mathbf{E}^{l}$ ist, indem wir noch die geforderten Eigenschaften von $\chi$ beachten. Unter Berufung auf das gleiche Literatur- 
zitat ist dann zu beliebiger Funktion $x \in C^{0, n}[0, T]$ die mengenwertige Abbildung $U(\cdot, x(\cdot))$ mit

$$
U(t, x(r))=\left\{v \in U_{0}(t) \mid \chi(t, x(t), v) \leqq 0\right\}
$$

normal auf $[0, T]$, da $\%(t, x(t), \cdot)$ eine stetige Funktion auf $\mathbf{E}^{l}$ und $\chi(\cdot, x(\cdot), v)$ eine meßbare Funktion auf $[0, T]$ ist $\forall v \in \mathbf{E}^{l}$. Damit ist Hilfssatz 5 bewiesen

Eine mengenwertige Abbildung $U$ gemäß Hilfssatz $\tilde{\boldsymbol{s}}$ genügt dann auch unseren Grundvoraussetzungen (2), da $U$ als normale mengenwertige Abbildung von $[0, T] \times \mathbf{E}^{n}$ in seiner Einschränkung auf die meßbare Teilmenge $\bar{G}$ wiederum normal ist. Außerdem sind ja alle $x \in W_{1}^{1, n}(0, T)$ zugleich Elemente des $C^{0, n}[0, T]$.

\section{Anhang II: Eine Erweiterung des Dualitätssatzes}

Nach [4] gilt zunächst ein Dualitätssatz der folgenden Gestalt:

$$
J_{0}(x, u) \geqq L(S):=\inf _{\epsilon \in \mathbb{M}_{0 . \xi} \in \mathbb{P}_{T}}\left[S\left(T, \xi_{T}\right)-S\left(0, \xi_{0}\right)\right]
$$

für alle $(x, u) \in \circlearrowleft$ und beliebige $S \in C^{1}(\bar{G})$, welche auf $\bar{G}$ den Nebenbedingungen

$$
S_{l}(t, \xi)+S_{\xi}{ }^{\prime}(\ell, \xi) g(t, \xi, v) \leqq f_{0}(t, \xi, v) \quad \forall v \in U(t, \xi)
$$

genügen. Nunmehr geben wir uns ein beliebiges $S$ vor, das den Voraussetzungen von Satz 1 genügt. Da $G$ 'ein Lipschitzgebiet ist, können wir dieses $S$ als Element des $W_{\infty}^{1}(G)$ stetig fortsetzen als Element des $W_{\infty}^{1}(\hat{G})$ zu einem umfassenderen Gebiet $\hat{G} \supset \supset G$ (vgl. dazu die Literaturangabe in Fußnote $\left.{ }^{1}\right)$ ). Damit können wir über die Sobolewschen Mittelfunktionen auf $\bar{G}$ eine Folge von Funktionen $S^{(k)} \in C^{1}(\bar{G})$ konstruieren, die folgende Eigenschaften besitzt: Die $S^{(k)}$ konvergieren für $k \rightarrow \infty$ auf $\bar{G}$ gleichmäßig gegen $S$ : die Folge der Gradienten $\nabla S^{(k)}$ konvergiert fast überall auf $G$ gleichmäßig gegen $\nabla S$. Infolgedessen existiert zu beliebig kleinem $\varepsilon>0$ ein $k_{0}(\varepsilon)$, so daß fast uiberall auf $G$

$$
S_{i}^{(k)}(t, \xi)+S_{\xi}^{(k)^{\prime}}\left(t,{ }^{\prime} \xi\right) g(t, \xi, v) \leqq f_{0}(t, \xi, v)+\varepsilon
$$

ist für alle $v \in U(t, \xi), k>k_{0}(\varepsilon)$. Indem wir die rechte Seite von (36) als neuen Integranden zu (1) auffassen, liefert unser Dualitätssatz (34)/(35) hierauf angewandt

$$
J_{0}(x, u)+\varepsilon T \geqq L\left(S^{(k)}\right) \quad \forall k>k_{0}(\varepsilon) .
$$

Nach Ausführung des Grenzübergangs $k \rightarrow \infty$ erhalten wir hieraus $J_{0}(x, u)+\varepsilon T$ $\geqq S_{T}-S_{0}$ und, da $\varepsilon>0$ beliebig klein vorgebbar war, schließlich $J_{0}(x, u) \geqq S_{T}-S_{0}$, womit Satz 1 bewiesen ist ${ }^{\prime}$

\section{IITERATUR}

[1] Ekgland, I., et R. Temam: Analyse convexe et problèmes variationnels. Paris 1974.

[2] GöpfFrt, A.: Mathematische Optimierung in allgemeinen Vektorräumen. Leipzig 1973.

[3] Иоффе, А. Д., и В. М. 'Тихомиров: Теория экстремальных задач. Москва 1974.

[4] KLötzLER, R.: On a general conception of duality in optimal control. In: Proceedings of the Conference EQUADIFF 4, Prague 1977, 189-196.

[5] KLötzler, R.: A generalization of the duality in optimal control. In: Procecdings of the 8th IFIP Conference.on Optimization Techniques, part I. Würzburg 1977, 313-320. 
[6] Klötzler, R.: Flow problems and duality. In: Banach-Center Publications. Warszaw 1983 (to appear).

[7] Ortlieb, C. P.: Dualität bei nichtkonvexen Stcuerungsproblemen. Preprint 78/17 d. Univ. Hamburg, Institut f. Angew. Math. (1978).

[8] Rockafellar, R. T.: Conjugate convex functions in optimal control and the calculus of variations. Journ. Math. Anal. Appl. 32 (1970), 174-222.

[9] VINTER, R. B., and R. M. LewTS: The equivalence of the strong and weak formulation for certain problems in optimal control. SIAM J. on Control and Optimization 16 (1978), $546-570$.

[10] Yodna, L. C.: Lectures on the calculus of variations and optimal control theory. Philadelphia 1969.

Manuskripteingang: 24.07. 1981

\section{VERF ASSER :}

Prof. Dr. Rolf Klötzler

Sektion Mathematik der Karl-Marx-Universität

DDR-7010 Leipzig, Karl-Marx-Platz 10 